\title{
Stochastic Spacecraft Trajectory Optimization with the Consideration of Chance Constraints
}

\author{
Runqi Chai, Member, IEEE, Al Savvaris, Member, IEEE, Antonios Tsourdos, Member, IEEE, Senchun Chai, and Yuanqing Xia, \\ Senior Member, IEEE,
}

\begin{abstract}
This paper investigates a computational framework based on optimal control for addressing the problem of stochastic trajectory optimization with the consideration of chance constraints. This design employs a discretization technique to parameterize uncertain variables and create the trajectory ensemble. Subsequently, the resulting discretized version of the problem is solved by applying standard optimal control solvers. In order to provide reliable gradient information to the optimization algorithm, a smooth and differentiable chance constraint approximation method is proposed to replace the original probability constraints. The established methodology is implemented to explore the optimal trajectories for a spacecraft entry flight planning scenario with noise-perturbed dynamics and probabilistic constraints. Simulation results and comparative studies demonstrate that the present chance constraint handling strategy can outperform other existing approaches analyzed in this study, and this computational framework can produce reliable and less conservative solutions for the chance-constrained stochastic spacecraft trajectory planning problem.
\end{abstract}

Index Terms-Optimal control, stochastic trajectory optimization, probability constraints, chance constraint handling strategy, spacecraft trajectory planning.

\section{INTRODUCTION}

$\mathbf{T}$ HE problem of generating optimal trajectories for spacecrafts, autonomous vehicles or robots has been an active research topic for the past several decades. This type of problem has been widelyresearched due to its extensive applications in industry and military fields [1]-[3]. Such applications include, for instance, unmanned aerial vehicles (UAV) rescue or observation missions [1], car racing tasks [2], and multiple autonomous vehicle deployment or motion planning [3]. Specifically, in [1] the authors calculated the timeoptimal trajectory for a quadrotor with the consideration of multiple obstacles and physical limitations of the quadrotor. A two-track car trajectory optimization model was considered in [2], wherein authors developed an optimal control-based virtual prototyping tool to calculate the fastest trajectory on a given track. Besides, Hausler et al. [3] solved an energy-optimal motion planning problem for multiple wheeled robots by designing a numerical optimization framework. Nevertheless, the core aim of this kind of problem is to determine a feasible path or trajectory, for a given vehicle/robot, to achieve a pre-specified target and optimize a predefined performance index. During the trajectory planning phase, a number of constraints should be also taken into account in order to achieve the mission-dependent requirements and protect the structural integrity.

The problem addressed in this research is an optimal flight trajectory design for a constrained spacecraft entry flight mission scenario. Numerous algorithms and strategies have been reported for solving this type of problem in the past decade [4], [5]. Among them, the development of optimal control theory-based trajectory

R. Chai, A. Savvaris and A. Tsourdos are with the School of Aerospace, Transport and Manufacturing, Cranfield University, UK, e-mail: (r.chai@cranfield.ac.uk), (a.savvaris@cranfield.ac.uk), and (a.tsourdos@cranfield.ac.uk).

S. Chai and Y. Xia are with the school of Automation, Beijing Institute of Technology, Beijing, China, e-mail: (chaisc97@163.com), (xia_yuanqing@bit.edu.cn). optimization techniques has received significant attentions [6], [7]. One important feature of using such an approach is that various mission requirements can be formulated as objectives or constraints and entailed in the optimization model. Contributions made to develop or apply this methodology can be found in the literature [8]-[10]. Misra and Bai [8] addressed a free-floating space-robotic trajectory planning problem using optimal control theory and convex quadratic optimization. In their work, the limits on the joint angles as well as the joint velocities were modeled as physical constraints and embedded in the optimization formulation. In addition, Pontani et al. [9] derived the necessary conditions for optimality of a spacecraft orbital maneuver problem based on the maximum principle, and calculated the optimal solution via a swarm optimization method. In their follow-up research [10], a two-loop optimal control structure based on a modified heuristic method was built so as to address a spacecraft interplanetary trajectory planning problem.

Although all the previously reported optimal control-based techniques have been shown to be effective and promising tools for generating optimal trajectories (in particular, optimal control sequences), they only target at deterministic models. It should be noted that in many real-world mission scenarios, various model or actuator uncertainties must frequently be considered during the trajectory planning phase. As a result, a proper treatment of the dynamics and constraints affected by stochastic variables is requested, which in turn brings the development of stochastic trajectory optimization [11][13].

With the introduction of stochastic variables, the vehicle dynamics should be modeled as an uncertain nonlinear system [14]-[17]. It is important to note that due to the nature of uncertain dynamics, some mission constraints such as the variable boundary conditions used in the deterministic trajectory optimization model can no longer be satisfied exactly. One way to handle these constraints is to rewrite them as robust constraints such that the calculated solution can satisfy these constraints with respect to any realization of the stochastic parameters [14], [18]. Another feasible strategy is to use probabilistic constraints or chance constraints [19]. Compared with the application of robust constraints, the use of chance constraints offers a number of advantages [20], [21]. Since this kind of strategy allows constraint violations to be less than a user-specified risk parameter, the optimization algorithms tend to have more flexibility to search optimal or nearoptimal solutions. Moreover, the feasible set defended by the chance constraints is usually larger than the one determined by its robust counterpart, which means the chance-constrained method is likely to be less conservative. Several instances regarding the use/development of chance constrained optimal control methods can be found in trajectory planning research [12], [22]. In [22], an autonomous vehicle chance-constrained trajectory generation problem was solved by performing a disjunctive convex optimization technique. However, if the system becomes nonlinear, this approach is no longer effective. Besides, the chance constraint handling strategy used in [22] tends to result in large conservatism. Furthermore, a chance-constrained stochastic optimal control framework was constructed and proposed by Zhao and Kumar in [12]. However, in their work, only the potential 
feasibility was illustrated but the effectiveness for solving chanceconstrained stochastic optimal control problems was not verified.

To overcome these issues and offer an effective alternative, we present a computational framework based on optimal control to address the problem of stochastic spacecraft trajectory optimization with the consideration of probabilistic constraints. So far to the best of the authors' knowledge, there are fewer results have been reported to generate the chance-constrained spacecraft flight trajectory. Consequently, the present investigation is an attempt to address this concern. The main contributions of this paper can be summarised into the following three aspects. First, a new computational optimal control framework that can be applied to solve stochastic chanceconstrained optimal control problems is designed. Second, in order to provide reliable gradient information to the optimization algorithm, a smooth and differentiable chance constraint approximation method is proposed to approximate the original probability constraints. By applying this chance constraint handling strategy, the overall framework can have the feasibility to be combined with standard optimal control solvers where gradient-based optimizers are used to generate the optimal solution. Third, a novel spacecraft entry flight planning mission scenario with noise-perturbed dynamics and probabilistic constraints is constructed and employed to verify the effectiveness of the proposed computational framework.

The structure of this paper is organised as follows. Section II presents the mathematical formulation of the chance-constrained stochastic optimal control problem. In Section III, the proposed computational framework as well as the chance constraint handling strategy is detailed. A new chance-constrained stochastic spacecraft entry trajectory planning model is demonstrated in Section IV. Section $\mathrm{V}$ presents a comprehensive simulation study. In Section VI, the concluding remark is drawn.

\section{Mathematical Formulation of the Problem}

The chance-constrained stochastic optimal control problems (CCSOCPs) to be considered through this investigation is established as follows:

$$
\begin{array}{cl}
\underset{u(t)}{\operatorname{minimize}} & J=\mathbb{E}\left[\Phi\left(x\left(t_{0}\right), t_{0}, x\left(t_{f}\right), t_{f}\right)\right. \\
& \\
\text { subject to } & \left.\quad+\int_{t_{0}}^{t_{f}} L(x(t), u(t), \xi) d t\right] \\
& \psi\left(x\left(t_{0}\right), t_{0}\right)=0 \\
& h(x(t), u(t), \xi)=0 \\
& g(x(t), u(t), \xi) \leq 0 \\
& \operatorname{Pr}\left\{\phi\left(x\left(t_{f}\right), t_{f}\right) \leq 0\right\} \geq \epsilon_{\phi} \\
& \operatorname{Pr}\{G(x(t), u(t), \xi) \leq 0\} \geq \epsilon_{G}
\end{array}
$$

where $\mathbb{E}(\cdot)$ denotes the expectation operator, whereas $\operatorname{Pr}(\cdot)$ is the probability. The performance index $J$ is defined in an expectation form, which can be described as Eq.(1a). Here, the term $\Phi$ denotes the terminal cost and the term $L$ is the process cost.

In the CCSOCP formulation, $x(t) \in \mathbb{R}^{n_{x}}$ and $u(t) \in \mathscr{U} \subset \mathbb{R}^{n_{u}}$ represent, respectively, the state and control variables defined on the time domain $t \in\left[t_{0}, t_{f}\right]$. Here, $\mathscr{U}$ is a compact set. $\xi$ stands for the uncertain variable which is assumed to have a known probability density function (PDF) $R(\xi)$ supported on $\Omega$, where $\Omega$ is a measurable open set (e.g. $\xi \in \Omega \subset \mathbb{R}^{n_{p}}$ ).

In Eq.(1), it is assumed that the functions $f, \phi$, and $G$ need at least to be measurable functions in $\xi$. Additionally, assuming an optimal solution $u^{*}(t)$ exists, the solution $x\left(t, x\left(t_{0}\right), u^{*}(t), \xi\right)$ needs to be a measurable function in $\xi$.

The stochastic dynamics of the system are then defined by the nonlinear function $f: \mathbb{R}^{n_{x}} \times \mathscr{U} \times \Omega \mapsto \mathbb{R}^{n_{x}}$. Besides, $h: \mathbb{R}^{n_{x}} \times \mathscr{U} \times \Omega \mapsto \mathbb{R}^{n_{h}}$ and $g: \mathbb{R}^{n_{x}} \times \mathscr{U} \times \Omega \mapsto \mathbb{R}^{n_{g}}$ are the stochastic equality and inequality constraints, respectively. $\psi$ : $\mathbb{R}^{n_{x}} \times \mathbb{R} \mapsto \mathbb{R}^{n_{\psi}}$ is the initial boundary condition. Eq.(1f) and Eq. $(1 \mathrm{~g})$ are referred to as chance constraints or probabilistic constraints with an acceptable probability of occurrence $\epsilon$. These two constraints can be explained that the valid state and control trajectories should fulfill the inequalities $\phi\left(x\left(t_{f}\right), t_{f}\right) \leq 0$ and $G(x(t), u(t), \xi) \leq 0$ with probability $\epsilon_{\phi}$ and $\epsilon_{G}$. The conditions for $\phi$ and $G$ are $\phi: \mathbb{R}^{n_{x}} \times \mathbb{R} \mapsto \mathbb{R}$ and $G: \mathbb{R}^{n_{x}} \times \mathscr{U} \times \Omega \mapsto \mathbb{R}$, respectively. Moreover, we have $\Phi: \mathbb{R}^{n_{x}} \times \mathbb{R} \times \mathbb{R}^{n_{x}} \times \mathbb{R} \mapsto \mathbb{R}$ and $L: \mathbb{R}^{n_{x}} \times \mathbb{U} \times \Omega \mapsto \mathbb{R}$. It is further supposed that the functions $L$, $f, h, g$ and $G$ are at least one-time continuously differential with respect to $(x, u, \xi) \in \mathbb{R}^{n_{x}} \times \mathscr{U} \times \Omega$.

Based on the CCSOCP formulation given by Eq.(1), the overall objective of this problem is to search the optimal control sequence $u(t)$ such that the performance index $J$ can be optimized subject to constraints (1b)-(1g).

\section{Methodology}

This section discusses the proposed computational optimal control framework that is applied to solve the CCSOCP formulation introduced in Section II. Firstly, an initial transformation of the chance constraints is introduced. Following that, the stochastic quadrature formulas are presented in Section III.B so as to discretize the uncertain variables. Then, the resulting discretized CCSOCP formulation is constructed in Section III.C to create the trajectory ensemble. In order to deal with the probabilistic constraints, a smooth and differentiable probabilistic constraint approximation strategy is employed in Section III.D. The chance constraint handling method, together with the created trajectory ensemble, is used to transcribe the original CCSOCP formulation into a discretized CCSOCP model which can be solvable for standard gradient-based optimal control solvers in Section III.E.

\section{A. Initial Transformation of Chance Constraints}

In problem (1), the chance constraints described by Eq.(1f) and Eq. $(1 \mathrm{~g})$ can be transformed to a more transparent form. Take Eq.(1g) as an example, the associated probability function can be defined by:

$$
P(u)=\operatorname{Pr}\{G(x, u, \xi) \leq 0\}=1-\operatorname{Pr}\{G(x, u, \xi)>0\}
$$

Considering the inequality $G(x, u, \xi) \leq 0$ as an event, the probability function $P(u)$ can be further written as

$$
P(u)=1-\mathbb{E}[H(G(x, u, \xi))]
$$

where $H(\cdot)$ denotes the unit jump function (also known as Heaviside function) with respect to $G(x, u, \xi)$ :

$$
H(G(x, u, \xi))=\left\{\begin{array}{lll}
1 & \text { if } & G(x, u, \xi) \geq 0 \\
0 & \text { if } \quad G(x, u, \xi)<0
\end{array}\right.
$$

Hence, Eq.(1f) and Eq.(1g) have the equivalent expressions:

$$
\begin{aligned}
& \mathbb{E}\left[H\left(\phi\left(x\left(t_{f}\right), t_{f}\right)\right)\right] \leq 1-\epsilon_{\phi} \\
& \mathbb{E}[H(G(x(t), u(t), \xi))] \leq 1-\epsilon_{G}
\end{aligned}
$$

\section{B. Stochastic Quadrature Formulas}

To solve the stochastic optimization problem (1), an important procedure is to approximate the uncertain variables appearing inside the dynamics and constraints. To do this, a certain stochastic quadrature formula (SQF) should be used to obtain the approximation with a desired error order (raft of convergence).

Definition 1. An SQF of degree $N$ can be regarded as a set of one-dimensional weighted parameters $\left\{w_{k}\right\}, k \in\{1,2, \ldots N\}$ and 
$n_{p}$-dimensional uncertain variables $\left\{\xi_{k}\right\}, k \in\{1,2, \ldots N\}, \xi_{k} \in \Omega$ such that the equation $\sum_{k=1}^{N} w_{k} f\left(\xi_{k}\right)=\int_{\Omega} f(\xi) d P(\xi)$ holds true. Here, $P$ is the probability measure. Supposing $R(\xi)$ is the PDF of $\xi$, it is obvious to get $d P(\xi)=R(\xi) d \xi . f \in L_{R}^{2}$, where $L_{R}^{2}$ is given by

$$
L_{R}^{2}=\left\{f: \Omega \mapsto \mathbb{R} \mid \int_{\Omega} f^{2}(\xi) R(\xi) d \xi<\infty\right\}
$$

With the introduction of SQF, an estimation of the stochastic integral can be built such that

$$
I=\mathbb{E}[G(\xi)]=\int_{\Omega} G(\xi) R(\xi) d \xi=\sum_{k=1}^{N} w_{k} G\left(\xi_{k}\right)
$$

It is worth noting that based on this approximation, one can easily obtain other statistical quantities. For example, the variance of $G(\xi)$ can be calculated by:

$$
\begin{aligned}
\operatorname{Var}(G(\xi)) & =\mathbb{E}\left[G^{2}(\xi)\right]-\mathbb{E}^{2}[G(\xi)] \\
& =\sum_{k=1}^{N} w_{k} G^{2}\left(\xi_{k}\right)-\left(\sum_{k=1}^{N} w_{k} G\left(\xi_{k}\right)\right)^{2}
\end{aligned}
$$

Until now, there are many effective SQF methods and their variances that have been reported for approximating the uncertain parameters. Among them, generalized polynomial chaos (gPC) theorybased approaches have attracted great attentions due to their ability in decomposing the stochastic variables into a convergent series of polynomials. gPC methods have been widely applied in various engineering applications [11], [23]. The general procedure of this $\mathrm{SQF}$ is to use deterministic orthogonal polynomials and coefficients for deriving the expression of stochastic systems. It was shown in [23] that the gPC-based techniques can be efficient for optimal control problems (OCPs) containing a relatively small number of stochastic variables. However, for the uncertain trajectory optimization problem given by Eq.(1), the uncertain effect in the dynamics and constraints must be considered during the entire time domain, which means the random variable will appear at each time instant. Specifically, if the uncertain dynamics contain $n_{p}$ random variables and $N_{j}$ number of temporal nodes are selected to discretize the time interval, the resulting number of uncertain variables becomes $N_{j} n_{p}$. Commonly, for practical trajectory planning problems that have a long time duration, a large $N_{j}$ will be chosen in order to produce desired solution accuracy. Consequently, for problem (1), gPC-based methods are no longer suitable for representing the uncertainty.

Another well-developed class of SQF techniques is the sampling-based methods. Typical examples include the Markov chain Monto Carlo (MCMC) approach and quasi-Monto Carlo methods. The motivation for the use of sampling-based methods relies on their simplicity and the fact that the approximation error order is independent with respect to the dimension of $\xi$. Take MCMC as an example, a stochastic variable ensemble $\{\xi\}_{k=1}^{N}$ can be constructed by randomly sampling from the probability distribution (e.g. $\{\xi\}_{k=1}^{N} \sim R(\xi)$ ). Each sample will be weighted equally (e.g. $\left.w_{k}=N^{-1}\right)$, thereby producing an $\mathcal{O}(1 / \sqrt{N})$ convergence rate in terms of the approximation error. Therefore, in this paper the MCMC technique is employed to model the uncertain parameters.

\section{Discretized CCSOCP Formulation}

Following the discussion stated in Section III.B, it is now assumed that an SQF technique is selected with a fixed number of $N$. This indicates that the uncertain variable ensemble $\left\{\xi_{k}\right\}_{k=1}^{N}$ will correspond to a trajectory ensemble $\left\{\left(x_{k}, u_{k}\right)\right\}_{k=1}^{N}$. In other words, each $\xi_{k}$, along with the initial condition $\psi\left(x_{k}\left(t_{0}\right), t_{0}\right)$, defines a unique trajectory and the trajectory ensemble is constructed by collecting all these trajectories. More precisely, the $k$ th trajectory is determined by the following equations:

$$
\left\{\begin{array}{l}
\dot{x}_{k}=f\left(x_{k}, u_{k}, \xi_{k}\right) \\
\psi\left(x_{k}\left(t_{0}\right), t_{0}\right)=0 \\
h\left(x_{k}, u_{k}, \xi_{k}\right)=0 \\
g\left(x_{k}, u_{k}, \xi_{k}\right) \leq 0 \\
\mathbb{E}\left[H\left(\phi\left(x_{k}\left(t_{f}\right), t_{f}\right)\right)\right] \leq 1-\epsilon_{\phi} \\
\mathbb{E}\left[H\left(G\left(x_{k}, u_{k}, \xi_{k}\right)\right)\right] \leq 1-\epsilon_{G}
\end{array}\right.
$$

A more compact form of the stochastic system (7) can be obtained by defining the augmented state, control and uncertain vectors described as follows:

$$
\begin{aligned}
& x_{A}=\left[x_{1}, x_{2}, \ldots, x_{k}, \ldots, x_{N}\right]^{T} \\
& u_{A}=\left[u_{1}, u_{2}, \ldots, u_{k}, \ldots, u_{N}\right]^{T} \\
& \xi_{A}=\left[\xi_{1}, \xi_{2}, \ldots, \xi_{k}, \ldots, \xi_{N}\right]^{T}
\end{aligned}
$$

Consequently, the functions $f, \psi, h$ and $g$ are rewritten as:

$$
\begin{gathered}
f_{A}\left(x_{A}, u_{A}, \xi_{A}\right) \\
=\left[\begin{array}{c}
f\left(x_{1}, u_{1}, \xi_{1}\right) \\
\vdots \\
f\left(x_{k}, u_{k}, \xi_{k}\right) \\
\vdots \\
f\left(x_{N}, u_{N}, \xi_{N}\right)
\end{array}\right], \quad=\left[\begin{array}{c}
\psi\left(x_{A}\left(t_{0}\right), t_{0}\right) \\
\psi\left(x_{1}\left(t_{0}\right), t_{0}\right) \\
\vdots \\
\psi\left(x_{k}\left(t_{0}\right), t_{0}\right) \\
\vdots \\
\psi\left(x_{N}\left(t_{0}\right), t_{0}\right)
\end{array}\right] \\
h_{A}\left(x_{A}, u_{A}, \xi_{A}\right) \\
=\left[\begin{array}{c}
h\left(x_{1}, u_{1}, \xi_{1}\right) \\
\vdots \\
h\left(x_{k}, u_{k}, \xi_{k}\right) \\
\vdots \\
h\left(x_{N}, u_{N}, \xi_{N}\right)
\end{array}\right], \quad=\left[\begin{array}{c}
g\left(x_{1}, u_{1}, \xi_{1}\right) \\
\vdots \\
g\left(x_{k}, u_{k}, \xi_{k}\right) \\
\vdots \\
g\left(x_{N}, u_{N}, \xi_{N}\right)
\end{array}\right]
\end{gathered}
$$

In terms of the objective function, based on the SQF and the constructed trajectory ensemble, one can rewrite the terminal cost as:

$$
\begin{aligned}
& \Phi_{A}\left(x_{A}\left(t_{0}\right), t_{0}, x_{A}\left(t_{f}\right), t_{f}\right) \\
& =\sum_{k=1}^{N} w_{k} \Phi\left(x_{k}\left(t_{0}\right), t_{0}, x_{k}\left(t_{f}\right), t_{f}\right)
\end{aligned}
$$

Analogically, the process cost term is expressed by:

$$
L_{A}\left(x_{A}, u_{A}, \xi_{A}\right)=\sum_{k=1}^{N} w_{k} L\left(x_{k}, u_{k}, \xi_{k}\right)
$$

Based on Eq.(10) and Eq.(11), the overall cost function of the discretized problem can be defined by

$$
J_{A}=\Phi_{A}\left(x_{A}\left(t_{0}\right), t_{0}, x_{A}\left(t_{f}\right), t_{f}\right)+\int_{t_{0}}^{t_{f}} L_{A}\left(x_{A}, u_{A}, \xi_{A}\right)
$$

Regarding the probabilistic boundary condition $\phi$ and path constraint $G$, two similar expressions are obtained, which can be given by:

$$
\begin{aligned}
& \mathbb{E}\left[H_{A}\left(\phi\left(x_{A}\left(t_{f}\right), t_{f}\right)\right)\right]=\sum_{k=1}^{N} w_{k} H\left(\phi\left(x_{k}\left(t_{f}\right), t_{f}\right)\right) \\
& \mathbb{E}\left[H_{A}\left(G\left(x_{A}, u_{A}, \xi_{A}\right)\right)\right]=\sum_{k=1}^{N} w_{k} H\left(G\left(x_{k}, u_{k}, \xi_{k}\right)\right)
\end{aligned}
$$

According to all the definitions and transformations given by Eqs.(8)-(13), the discretized version of the original CCSOCP formu- 
lation can be written as:

$$
\begin{array}{cl}
\underset{u_{A}}{\operatorname{minimize}} & J_{A}=J_{\Phi}+J_{L} \\
\text { subject to } & \dot{x}_{A}=f_{A}\left(x_{A}, u_{A}, \xi_{A}\right) \\
& \psi_{A}\left(x_{A}\left(t_{0}\right), t_{0}\right)=0 \\
& h_{A}\left(x_{A}, u_{A}, \xi_{A}\right)=0 \\
& g_{A}\left(x_{A}, u_{A}, \xi_{A}\right) \leq 0 \\
& \mathbb{E}\left[H_{A}\left(\phi\left(x_{A}\left(t_{f}\right), t_{f}\right)\right)\right] \leq 1-\epsilon_{\phi} \\
& \mathbb{E}\left[H_{A}\left(G\left(x_{A}, u_{A}, \xi_{A}\right)\right)\right] \leq 1-\epsilon_{G}
\end{array}
$$

where $J_{\Phi}$ and $J_{L}$ are the abbreviations of the first and second terms of Eq.(12). This discretized formulation will be applied as an approximant to the original CCSOCP problem.

Remark 1. According to the sampled approximation equations, the hard constraints are approximated by requiring that the constraints are satisfied for all sampled disturbance realizations. This might result in some approximation errors. In fact, depending on the number of samples, stochastic bounds for the probability of constraint violation can be derived [24]. Take $h(\cdot)$ as an example, by introducing two constants $c_{1}$ and $c_{2}$, we have:

$$
\left|\int_{\Omega} h(\xi) R(\xi) d \xi-\sum_{k=1}^{N} w_{k} h\left(\xi_{k}\right)\right| \geq c_{1}\|h\| N^{-1}
$$

Moreover, the mean square bound can be written as:

$$
\left\{\mathbb{E}\left[\int_{\Omega} h(\xi) R(\xi) d \xi-\sum_{k=1}^{N} w_{k} h\left(\xi_{k}\right)\right]^{2}\right\}^{1 / 2} \geq c_{2}\|h\| N^{-1 / 2}
$$

Here, $c_{1}$ and $c_{2}$ are implicitly depended on $\xi$.

\section{Chance Constraint Handling Strategy}

In this investigation, we are interested in applying standard optimal control solvers to optimize the state and control trajectories. However, the discretized CCSOCP formulation shown in Eq.(14) is not solvable in its present form. This is because the evaluation of Eq.(14f) and Eq.(14g) does not provide reliable gradient information. It is important to remark that for most typical optimal control solvers, gradient-based optimization strategies are used to search the optimal decision variables. As a result, due to the requirement of derivative information, it is desired to find a smooth and differentiable approximation for replacing the chance constraints (e.g. Eq.(14f) and Eq. $(14 \mathrm{~g}))$.

In order to remove any ambiguous annotations, the general expectation form of chance constraints (shown in Eq.(5)) is recalled in the following analysis. Taking Eq.(5b) as an instance, as can be seen from this equation, the approximation of $\mathbb{E}[H(G(x, u, \xi))]$ eventually becomes finding an approximation of $H(G(x, u, \xi))$. More precisely, the key idea of the approximation is to design a function $\Psi(c, G(x, u, \xi)):[1, \infty) \times \mathbb{R} \mapsto \mathbb{R}$ such that $\Psi(c, G(x, u, \xi))$ is upper bounded and strictly greater than the unit jump function $H(G(x, u, \xi))$ (e.g. $H(G(\cdot, \cdot, \cdot))<\Psi(c, G(\cdot, \cdot, \cdot)) \leq C<\infty$, where $C$ is a positive constant). If $\Psi(c, G(x, u, \xi))$ is employed to replace $H(G(x, u, \xi))$, then the associated probability function is changed to

$$
P(u) \geq 1-\mathbb{E}[\Psi(c, G(x, u, \xi))]
$$

The corresponding feasible set associated with the chance constraint should be defined as:

$$
\mathscr{F}(c):=\left\{x \in \mathbb{R}^{n_{x}}, u \in \mathscr{U} \mid \mathbb{E}(\Psi(c, G(x, u, \xi))) \leq 1-\epsilon_{G}\right\}
$$

It is obvious that any $(x, u) \in \mathscr{F}(c)$ can be feasible for the original chance constraint, which means $\mathscr{F}(c)$ is a subset of

$$
\mathscr{O}:=\left\{x \in \mathbb{R}^{n_{x}}, u \in \mathscr{U} \mid \mathbb{E}(H(G(x, u, \xi))) \leq 1-\epsilon_{G}\right\}
$$

Several approximation functions were reported and verified in the literature. For instance, in [25] a modified exponential function in the form of $\Psi_{1}(c, G(x, u, \xi))=e^{\frac{1}{c} G(x, u, \xi)}, c=[0, \infty)$ was analyzed. Besides, a function $\Psi_{2}(c, G(x, u, \xi))=q_{d}(G(x, u, \xi))$ was proposed and verified in [26]. In $\Psi_{2}, q_{d}(\cdot)$ is the solution of a polynomial optimization problem. $d$ is the degree of the polynomial and $c=1 / d . \Psi_{1}$ and $\Psi_{2}$ were shown to be upper approximations of $H$. However, for $\Psi_{1}$, the corresponding feasible set is relatively small, thereby producing poor solution optimality. As for $\Psi_{2}$, when $G(x, u, \xi)$ has strong nonlinearities, the polynomial optimization problem becomes hard to solve. To avoid these problems and provide an effective alternative, in this study we suggest an approximation function in the following form:

$$
\Psi(c, G(x, u, \xi))=\frac{c+m_{1}}{c+m_{2} e^{-c G(x, u, \xi)}}
$$

where $c \in[1, \infty)$ is a control parameter; $m_{1}$ and $m_{2}$ are two positive constants with the relationship $m_{1} \leq m_{2}$. Based on Eq.(17), the derivative of $\Psi(c, G(x, u, \xi))$ with respect to $G$ can be evaluated by:

$$
\begin{aligned}
\mu(c, G(x, u, \xi)) & =\frac{\partial}{\partial G(x, u, \xi)} \Psi(c, G(x, u, \xi)) \\
& =\frac{c\left(c+m_{1}\right)\left(c+m_{2} e^{-c G(x, u, \xi)}\right)}{\left(c+m_{2} e^{-c G(x, u, \xi)}\right)^{2}}
\end{aligned}
$$

The approximation function (17) has several properties:

(a) $\Psi(c, G(x, u, \xi))$ is positive for any value of $G(x, u, \xi)$.

(b) If $G(x, u, \xi) \geq 0$, then $\Psi(c, G(x, u, \xi)) \geq 1$.

(c) $\Psi(c, \cdot)$ is a monotonically increasing function with respect to $G(x, u, \xi)$.

(d) $\Psi(c, G(x, u, \xi))$ tends to become closer to $H(G(x, u, \xi))$ as the control parameter $c$ increases.

Properties (a)-(c) guarantee that $\Psi(c, G(x, u, \xi))$ is a strict upper bound of $H(G(x, u, \xi))$. To better show Property (d), a figure illustrating $\Psi(c, G(x, u, \xi))$ and $\Psi_{1}(c, G(x, u, \xi))$ approximations is plotted (see Fig.1).

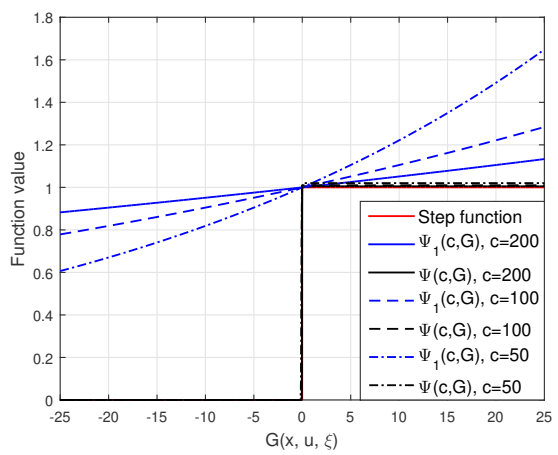

Fig. 1: Different approximation functions

Obviously, the approximation accuracy obtained by applying $\Psi(c, G(x, u, \xi))$ becomes higher as $c$ increases, thus making this approximation less conservative.

\section{E. Solvable CCSOCP Model}

Applying the chance constraint handling strategy developed in Section III.D, Eq.(13) can be further transformed to

$$
\begin{aligned}
\mathbb{E}\left[H_{A}\left(\phi\left(x_{A}\left(t_{f}\right), t_{f}\right)\right)\right] & <\mathbb{E}\left[\Psi_{A}\left(c, \phi\left(x_{A}\left(t_{f}\right), t_{f}\right)\right)\right] \\
& =\sum_{k=1}^{N} w_{k} \Psi\left(c, \phi\left(x_{k}\left(t_{f}\right), t_{f}\right)\right) \\
\mathbb{E}\left[H_{A}\left(G\left(x_{A}, u_{A}, \xi_{A}\right)\right)\right] & <\mathbb{E}\left[\Psi_{A}\left(c, G\left(x_{A}, u_{A}, \xi_{A}\right)\right)\right] \\
& =\sum_{k=1}^{N} w_{k} \Psi\left(c, G\left(x_{k}, u_{k}, \xi_{k}\right)\right)
\end{aligned}
$$


Hence, the derivative of Eq.(19b) with respect to the state and control variables can be calculated by:

$$
\begin{aligned}
& \sum_{k=1}^{N} w_{k} \nabla_{x_{k}} \Psi\left(c, G\left(x_{k}, u_{k}, \xi_{k}\right)\right) \\
& =\left.\sum_{k=1}^{N}\left[\frac{\partial}{\partial s} \Psi(c, s) \nabla_{x_{k}} G\left(x_{k}, u_{k}, \xi_{k}\right)\right]\right|_{s=G\left(x_{k}, u_{k}, \xi_{k}\right)}
\end{aligned}
$$

and

$$
\begin{aligned}
& \sum_{k=1}^{N} w_{k} \nabla_{u_{k}} \Psi\left(c, G\left(x_{k}, u_{k}, \xi_{k}\right)\right) \\
& =\left.\sum_{k=1}^{N}\left[\frac{\partial}{\partial s} \Psi(c, s) \nabla_{u_{k}} G\left(x_{k}, u_{k}, \xi_{k}\right)\right]\right|_{s=G\left(x_{k}, u_{k}, \xi_{k}\right)}
\end{aligned}
$$

where $\left.\frac{\partial}{\partial s} \Psi(c, s)\right|_{s=G\left(x_{k}, u_{k}, \xi_{k}\right)}$ is calculated according to Eq.(18).

Combining Eq.(14) and Eq.(19), the updated CCSOCP model which is solvable for standard gradient-based optimal control solvers can be established. This solvable version is demonstrated in Eq.(22).

$$
\begin{array}{cl}
\underset{u_{A}}{\operatorname{minimize}} & J_{A}=J_{\Phi}+J_{L} \\
\text { subject to } & \dot{x}_{A}=f_{A}\left(x_{A}, u_{A}, \xi_{A}\right) \\
& \psi_{A}\left(x_{A}\left(t_{0}\right), t_{0}\right)=0 \\
& h_{A}\left(x_{A}, u_{A}, \xi_{A}\right)=0 \\
& g_{A}\left(x_{A}, u_{A}, \xi_{A}\right) \leq 0 \\
& \sum_{k=1}^{N} w_{k} \Psi\left(c, \phi\left(x_{k}\left(t_{f}\right), t_{f}\right)\right) \leq 1-\epsilon_{\phi} \\
& \sum_{k=1}^{N} w_{k} \Psi\left(c, G\left(x_{k}, u_{k}, \xi_{k}\right)\right) \leq 1-\epsilon_{G}
\end{array}
$$

\section{Chance-Constrained Stochastic Spacecraft Entry Trajectory Planning: System Modeling}

In this section, an application of the proposed computational framework to the problem of spacecraft trajectory planning is presented. A time-optimal spacecraft entry trajectory optimization problem studied in [27] is further extended by considering the uncertainties involved in the vehicle dynamics, terminal state conditions, and control actuation. These uncertainties are modeled into stochastic dynamics and chance constraints, which are then entailed in the optimization model and adopted to search the optimal state and control profiles. Therefore, to solve the problem, the CCSOCP optimization model associated with it should be firstly constructed.

\section{A. Stochastic Dynamics and Objective Function}

Prior to introducing in detail the stochastic optimization model, it is worth recalling some backgrounds of this mission scenario. The spacecraft re-enters the atmosphere at a pre-specified position and descends down to an target altitude point for observation and gathering of information of inaccessible areas [27]. Once the observation mission is completed, the vehicle starts the ascending phase, existing the atmosphere and returning back to the original orbit. Since most path constraints are likely to be violated during the descending, the most challenging entry phase is considered in this study. The dynamics of the spacecraft are, therefore, given by the following system of stochastic differential equations:

$$
\dot{x}=\frac{d}{d t}\left[\begin{array}{c}
r \\
\theta \\
\varphi \\
V \\
\gamma \\
\chi
\end{array}\right]=\left[\begin{array}{c}
V \sin \gamma+\xi_{r} \\
\frac{V \cos \gamma \sin \chi}{r \cos \varphi}+\xi_{\theta} \\
\frac{V \cos \gamma \cos \chi}{r}+\xi_{\varphi} \\
-\frac{D(\alpha)}{m}-g \sin \gamma+\xi_{V} \\
\frac{L(\alpha) \cos \sigma}{m V}+\left(\frac{V^{2}-g r}{r V}\right) \cos \gamma+\xi_{\gamma} \\
\frac{L(\alpha) \sin \sigma}{m V \cos \gamma}+\frac{V}{r} \cos \gamma \sin \chi \tan \varphi+\xi_{\chi}
\end{array}\right]
$$

where $x=[r, \theta, \varphi, V, \gamma, \chi]^{T}$ are the state variables representing the radial distance, longitude, latitude, speed, flight path angle (FPA) and azimuth angle, respectively. The control variables are composed by the angle of attack $\alpha$ (AOA) and bank angle $\sigma \cdot m$ denotes the vehicle's mass, whereas $g$ stands for the gravity acceleration. $D(\alpha)$ and $L(\alpha)$ are the drag and lift forces and they are functions of AOA. $\xi_{x}=\left[\xi_{r}, \xi_{\theta}, \xi_{\varphi}, \xi_{V}, \xi_{\gamma}, \xi_{\chi}\right]^{T}$ are the uncertain disturbances caused by error modeling or varying flight conditions. For brevity, this uncertain system is abbreviated as $\dot{x}=f(x, u)+\xi_{x}$.

Since it is desired to fulfill the entry mission in the shortest time, minimizing the final time instant is chosen as the objective function $J=\min t_{f}$.

Remark 2. Different from the dynamics used in our previous work [28], the vehicle's engine model is dropped out from Eq.(23), which means the spacecraft only uses the aerodynamic forces to manoeuver during the atmospheric flight. Although the vehicle can fire its engine so as to achieve a shorter time duration, it was found in [29] that this design tends to result in a significant mass fraction and the spacecraft might have no fuel to carry out the continuing mission. Therefore, it is suggested to use the aero-assisted model given by Eq.(23) during the atmospheric entry flight.

\section{B. Hard Constraints and Chance Constraints}

In the stage of entry flight, a number of limitations should be taken into account in the design of optimal flight paths. The first constraint is to limit the angular rate of control variables such that the control sequence and its derivative cannot vary significantly. To achieve this, two rate constraints are formulated, which can be illustrated by:

$$
\left\{\begin{array} { l } 
{ \dot { \alpha } = k _ { \alpha } ( \alpha _ { c } - \alpha ) } \\
{ \dot { \sigma } = k _ { \sigma } ( \sigma _ { c } - \sigma ) }
\end{array} \quad \left\{\begin{array}{l}
\alpha_{c} \in\left[\alpha_{c}^{\min }, \alpha_{c}^{\max }\right] \\
\sigma_{c} \in\left[\sigma_{c}^{\min }, \sigma_{c}^{\max }\right]
\end{array}\right.\right.
$$

where $\left[\alpha_{c}^{\min }, \alpha_{c}^{\max }\right]$ and $\sigma_{c} \in\left[\sigma_{c}^{\min }, \sigma_{c}^{\max }\right]$ define the allowable regions of the controls. As can be observed from Eq.(24), the control variable now becomes the demanded AOA $\alpha_{c}$ and bank angle $\sigma_{c}$. Eq.(24) is adhered to Eq.(23), thus increasing the state space order by two.

To protect the structure of the spacecraft, several path constraints should also be considered in the optimization process. These requirements are required to satisfy during the entire time history and can be expressed as:

$$
g(x, u)=\left[\begin{array}{c}
\dot{Q}(x, u) \\
P_{d}(x, u) \\
n_{L}(x, u)
\end{array}\right]=\left[\begin{array}{c}
K_{Q} \rho^{0.5} V^{3} \\
\frac{1}{2} \rho V^{2} \\
\frac{\sqrt{L^{2}+D^{2}}}{m g}
\end{array}\right] \leq\left[\begin{array}{c}
\dot{Q}^{\max } \\
P_{d}^{\max } \\
n_{L}^{\max }
\end{array}\right]
$$

in which $\dot{Q}, P_{d}$ and $n_{L}$ are, respectively, the heat flux, dynamic pressure and normal acceleration. Their maximum allowable values are given by $\left[\dot{Q}^{\max }, P_{d}^{\max }, n_{L}^{\max }\right]^{T}$.

For the deterministic version of this problem [27], [28], one objective is required for the spacecraft to strike the the pre-specified terminal boundary conditions (e.g. $x_{f}=x\left(t_{f}\right)=\left[r_{f}, \gamma_{f}\right]$ ). However, when the problem is extended to the stochastic version, this requirement might not be achieved exactly due to the nature of stochastic dynamics. Therefore, the terminal state chance constraints are applied such that the final state variables can be restricted to a region of $x_{f}=\left[r_{f}, \gamma_{f}\right]$ with a prescribed violation rate value.

$$
\left\{\begin{array}{l}
\operatorname{Pr}\left(\left|r\left(t_{f}\right)-r_{f}\right| \leq \delta_{1}\right) \geq \epsilon_{1} \\
\operatorname{Pr}\left(\left|\gamma\left(t_{f}\right)-\gamma_{f}\right| \leq \delta_{2}\right) \geq \epsilon_{2}
\end{array}\right.
$$

where $\delta_{1}$ and $\delta_{2}$ are the maximum allowable deviation between the actual terminal state values and the pre-determined final state values. $1-\epsilon_{1}$ and $1-\epsilon_{2}$ are the corresponding risk values.

Similarly, in practice, the maximum attainable control actuation of the spacecraft may not be fixed. and is usually influenced by some 
uncertainties. These effects are modeled as probabilistic constraints which can be described as:

$$
\left\{\begin{array}{l}
\operatorname{Pr}\left(\alpha_{c}+\xi_{\alpha} \leq \alpha_{c}^{\max }\right) \geq \epsilon_{\alpha} \\
\operatorname{Pr}\left(\sigma_{c}+\xi_{\sigma} \leq \sigma_{c}^{\max }\right) \geq \epsilon_{\sigma}
\end{array}\right.
$$

in which $\xi_{\alpha}$ and $\xi_{\sigma}$ are two uncertain variables associated with the demanded AOA and band angle, respectively. $\epsilon_{\alpha}$ and $\epsilon_{\sigma}$ are the acceptable probability of occurrence.

\section{Simulation StUdy AND ANALYsis}

\section{A. Parameter Specification}

This section simulates the chance-constrained stochastic spacecraft entry trajectory planning problem modeled in Section IV by applying the methodology developed in Section III. The variable initial boundary values are assigned as: $x_{0}=$ $[80 \mathrm{~km}, 0 \mathrm{deg}, 0 \mathrm{deg}, 7802.9 \mathrm{~m} / \mathrm{s},-1 \mathrm{deg}, 90 \mathrm{deg}]^{T}$. Other missiondependent/vehicle-dependent variables used for the experiments are tabulated in Table.I.

TABLE I: Parameters used in the experiments

\begin{tabular}{lclc}
\hline \hline Parameters & Values/ranges & Parameters & Values/ranges \\
\hline Altitude, $h$ & {$[80 \mathrm{~km}, 50 \mathrm{~km}]$} & Azimuth, $\chi$ & {$\left[-90^{\circ}, 90^{\circ}\right]$} \\
Longitude, $\theta$ & {$\left[0^{\circ}, 90^{\circ}\right]$} & Mass, $m$ & $89160 \mathrm{~kg}$ \\
Latitude, $\varphi$ & {$\left[0^{\circ}, 90^{\circ}\right]$} & AOA, $\alpha$ & {$\left[0^{\circ}, 40^{\circ}\right]$} \\
Speed, $V$ & {$[3 \mathrm{~km} / \mathrm{s}, 8 \mathrm{~km} / \mathrm{s}]$} & Bank angle, $\sigma$ & {$\left[-90^{\circ}, 1^{\circ}\right]$} \\
FPA,$\gamma$ & {$\left[-10^{\circ}, 10^{\circ}\right]$} & $\alpha_{c}$ & {$\left[0^{\circ}, 40^{\circ}\right]$} \\
$\sigma_{c}$ & {$\left[-90^{\circ}, 1^{\circ}\right]$} & $\dot{Q}^{\max }$ & $160 \mathrm{BTU}$ \\
$P_{d}^{\max }$ & $13406.46 \mathrm{~Pa}$ & $n_{L}^{\max }$ & 2.5 \\
\hline \hline
\end{tabular}

In terms of the uncertain model, the stochastic dynamics is set in the form of $\dot{x}=f(x, u)+\xi_{x} f(x, u)$, where $\xi_{x}$ is supposed to have a normal distribution (e.g. $\xi_{x} \sim N\left(0,0.1^{2}\right)$ ). The uncertain term $\xi_{x} f(x, u)$ is augmented to the nominal dynamics and $\xi_{x}$ determines the magnitude of the model error. Besides, the vehicle's mass is also considered as an uncertain variable and is perturbed uniformly up to $2.5 \%$.

As for the control actuation and terminal state chance constraints, their approximate form which is illustrated in Eq.(28) can be obtained based on Eq.(19).

$$
\left\{\begin{array}{l}
\mathbb{E}_{\xi_{1}}\left(\Psi\left(c,\left|r\left(t_{f}\right)-r_{f}\right|-\delta_{1}\right)\right) \leq 1-\epsilon_{1} \\
\mathbb{E}_{\xi_{2}}\left(\Psi\left(c,\left|\gamma\left(t_{f}\right)-\gamma_{f}\right|-\delta_{2}\right)\right) \leq 1-\epsilon_{2} \\
\mathbb{E}_{\xi_{\alpha}}\left(\Psi\left(c, \alpha_{c}+\xi_{\alpha}-\alpha_{c}^{\max }\right)\right) \leq 1-\epsilon_{\alpha} \\
\mathbb{E}_{\xi_{\sigma}}\left(\Psi\left(c, \sigma_{c}+\xi_{\sigma}-\sigma_{c}^{\max }\right)\right) \leq 1-\epsilon_{\sigma}
\end{array}\right.
$$

In Eq.(28), the acceptable probabilities of occurrence are set as: $\epsilon_{1}=\epsilon_{2}=0.95$, and $\epsilon_{\alpha}=\epsilon_{\sigma}=0.90$. The target final conditions are $\left[r_{f}, \gamma_{f}\right]=[50 \mathrm{~km}, 0 \mathrm{deg}]$, while $\left[\delta_{1}, \delta_{2}\right]=[300 \mathrm{~m}, 0.02 \mathrm{deg}]$. The uncertain variables $\xi_{\alpha}$ and $\xi_{\sigma}$ are supposed to have exponential distributions, whose PDFs are given by $f(x, \lambda)=\lambda e^{\lambda x}, x \geq 0$ with the rate parameter $\lambda=1.5$. The sample size as well as the control parameters with respect to the chance constraints handling method are assigned as $N=2 \times 10^{5}, m_{1}=1.0, m_{2}=0.5$ and $c=10000$, respectively.

Following the transformation process discussed in Section III, the original chance-constraint stochastic spacecraft entry trajectory problem is reformulated to a deterministic CCSOCP, which can be solved by standard optimal control solvers. Currently, there are many possible optimization solvers such as the heuristic-based optimizer [9], [10] and the interior point-based optimizer [4], [19]. In this investigation, all the experiments were carried out by performing a newly-developed hybrid optimal control solver reported in [28]. The motivation of the use of this specific optimizer relies on its improved convergence ability in finding optimal solutions under perturbed environment.

\section{B. Performance of the Chance Constraint Handling Strategy}
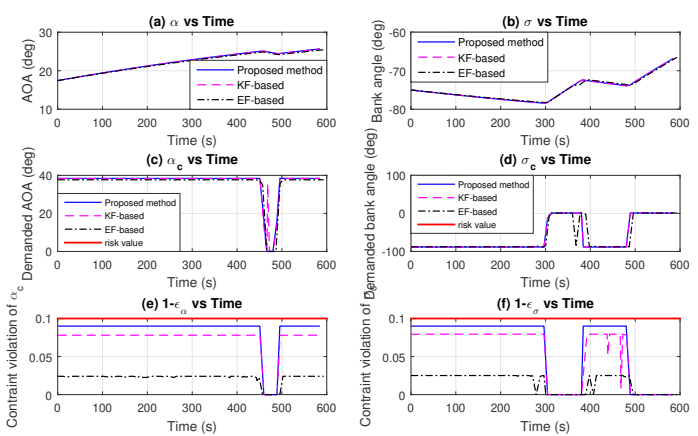

Fig. 2: Optimal AOA results obtained using different methods

The performance of the chance constraint handling strategy is tested and verified in this subsection. The negative effects caused by the noise-perturbed dynamic model are firstly eliminated. That is, the spacecraft entry problem is considered to find the chance-constrained optimal control profiles of the deterministic dynamical system. The optimized control solutions of the chance-constrained problem are depicted in Fig.2 and Fig.3. Specifically, Fig.2(a) and Fig.2(b) present the evolution of the AOA and bank angle, whereas Fig.2(c) and Fig.2(d) depict the demanded AOA and bank angle commands. In addition, the control actuation chance constraint violation trajectories are plotted in Fig.2(e) and Fig.2(f), where the permissable risk value (e.g. $1-\epsilon$ ) is indicated by the red line.

It should be noted that for the mission scenario considered in this study, it can be expected for the control variables to have a bang-bang behaviour. This is because the demanded controls are not involved in the path constraints explicitly, which means the optimal control sequence might contain corners and will switch between the allowable boundary values. This conclusion can be validated via the Proposition 3 derived in [28]. As can be observed from Fig.2, although the chance-constrained solutions are able to keep a switching structure, the magnitude cannot reach the allowable boundary values exactly. This can be explained that under the consideration of actuator uncertainty, $u^{*}(t)$ tends to be smaller than the allowable value. According to the results shown in Fig.2(e) and Fig.2(f), the chance constraint handling strategy is able to produce optimal solutions without violating the probabilistic constraints, which confirms that the effectiveness of this approach can be guaranteed.

A comparative simulation was carried out to compare the constraint violation histories achieved by performing the present technique and other typical strategies. For example, an exponential function-based approach (denoted as EF-based) investigated in [25], and a kinship function-based method (denoted as KF-based) reported in [26]. The results of these two approaches are plotted in Fig.2, from where it can be seen that compared with other methods, the method investigated in this study can produce a violation rate history that is closer to the pre-assigned risk value. Furthermore, the objective function value associated with it is $J^{*}=584.73 \mathrm{~s}$, which is again more optimal than that of the EF-based approach with $J_{E F}=592.99 \mathrm{~s}$ and KF-based method with $J_{K F}=586.31 \mathrm{~s}$.

\section{Sensitivity With Respect to Control Parameter c}

An attempt is made to analyze the sensitivity of the control parameter $c$ with respect to the chance constraint handling strategy. This sensitivity analysis has been performed by taken into account several variations of $c$ (e.g. $c_{1}=200, c_{2}=500, c_{3}=1000, c_{4}=$ 
TABLE II: Maximum violation rate achieved (10\% allowable)

\begin{tabular}{lccccc}
\hline \hline Sample size $N$ & $N_{1}$ & $N_{2}$ & $N_{3}$ & $N_{4}$ & $N_{5}$ \\
\hline \hline$c=10000$ & $9.04 \%$ & $9.04 \%$ & $9.07 \%$ & $9.11 \%$ & $9.01 \%$ \\
$c=5000$ & $7.79 \%$ & $7.81 \%$ & $7.81 \%$ & $7.76 \%$ & $7.84 \%$ \\
$c=2000$ & $6.50 \%$ & $6.36 \%$ & $6.22 \%$ & $6.42 \%$ & $6.45 \%$ \\
$c=1000$ & $4.59 \%$ & $4.39 \%$ & $4.27 \%$ & $4.39 \%$ & $4.37 \%$ \\
$c=500$ & $2.17 \%$ & $2.32 \%$ & $2.23 \%$ & $2.25 \%$ & $2.45 \%$ \\
$c=200$ & $0.31 \%$ & $0.33 \%$ & $0.15 \%$ & $0.29 \%$ & $0.21 \%$ \\
\hline \hline
\end{tabular}
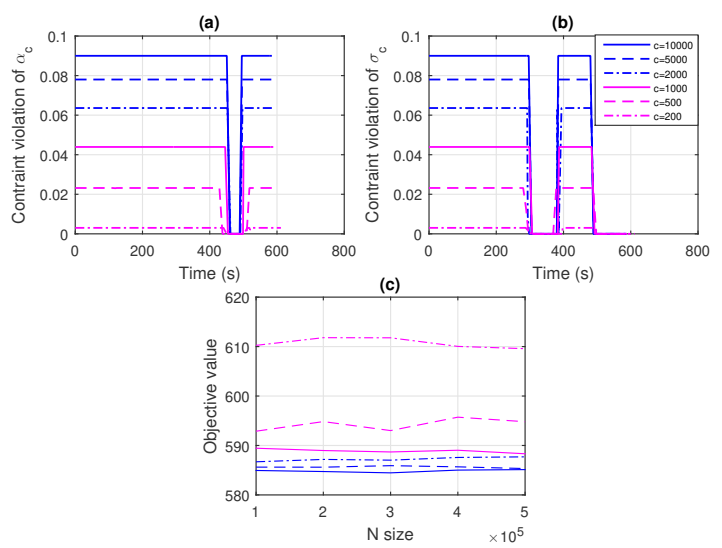

Fig. 3: Sensitivity results with respect to $c$ and $N$
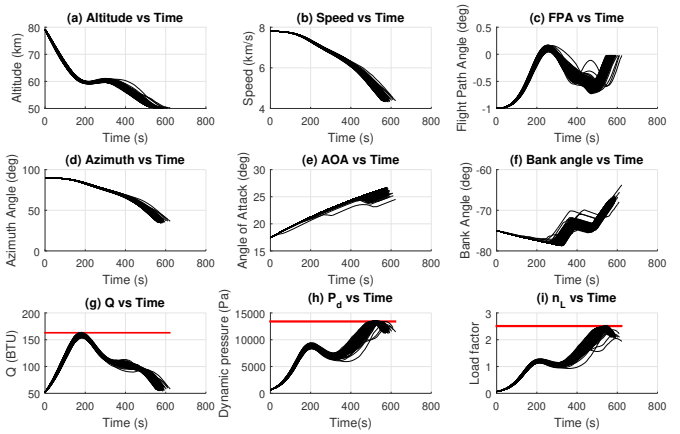

Fig. 4: State and path constraint results (500 dispersed trajectories)

$2000, c_{5}=5000$ and $c_{6}=10000$, respectively). The constraint violation histories achieved with different $c$ are shown in Fig.3(a) and Fig.3(b).

From Fig.3(a) and Fig.3(b), it is obvious that the optimal solution tends to be sensitive with respect to the selection of the control parameter $c$. Specifically, the obtained objective values are $J_{1}^{*}=611.80, J_{2}^{*}=594.83, J_{3}^{*}=588.98, J_{4}^{*}=587.18$, $J_{5}^{*}=585.61$ and $J_{6}^{*}=584.73$, respectively. A more aggressive constraint violation history can be achieved by increasing the value of $c$. This follows the discussion stated in Section III. However, based on our experiments, it was found that a large $c$ might result in numerical difficulties for the nonlinear programming (NLP) solver. Since $c$ does not contain any physical meaning, it is usually hard to select a proper $c$ that can balance the computational difficulty and the solution accuracy. This paper applies a fixed $c=10000$ to generate the all the solutions. However, an adaptive strategy should be designed in the follow-up research.

\section{Sensitivity With Respect to Sample Size N}

It should be noted that another important factor that might influence the solution accuracy is the sample size $N$. Hence a sensitivity study of the sample size $N$ to the chance constraint approximation method has also been carried out. By setting $N_{1}=1 \times 10^{5}$, $N_{2}=2 \times 10^{5}, N_{3}=3 \times 10^{5}, N_{4}=4 \times 10^{5}$ and $N_{5}=5 \times 10^{5}$ for different values of $c$, the solutions are generated and shown in Fig.3(c) and Table.II.

Fig.3(c) illustrates the results on the objective function value versus the number of sample $N$ plane, while Table.II summarised the maximal violation rate values achieved for different cases. From Fig.3(c) and Table.II, it can be observed that for a fixed control parameter $c$, the objective value does not vary significantly as the sample number $N$ increases. A similar behaviour can also be found in terms of the maximum violation rate. Therefore, it can be concluded that compared with the sample size $N$, the optimal results tend to be more sensitive with respect to the control variable $c$ of the developed method for solving the chance-constrained spacecraft entry trajectory planning problem.

Remark 3. It is important to note that although Fig.3(c) and Table.II display a less sensitive behaviour of $N$, this is only valid for the example considered in this paper and a counterexample can be easily constructed. Actually, since the Monte-Carlo sampling is known to have slow convergence, if a higher risk value is desired for the problem, a relatively large number of realization is usually required.

\section{E. Optimal Trajectories for the Stochastic Entry Problem}

From the previous subsections, it can be concluded that the chance constraint handling strategy studied in this research is reliable to produce more aggressive allowable rate values, which in turn offers more optimality of the solutions. As a result, the newly-developed technique for chance constraints is applied to solve the spacecraft CCSOCP for noise-perturbed dynamic systems. Fig. 4 presents the trajectories of the state variables and path constraints. Fig.5 displays the time evolution of the demanded control variables as well as the chance constraint violation rate. Each single line denotes the trajectory corresponding to an ensemble number $k$, and it is worth noting that in order to preserve the quality of the figure, only 500 dispersed trajectories are presented in Fig.4 and Fig.5.
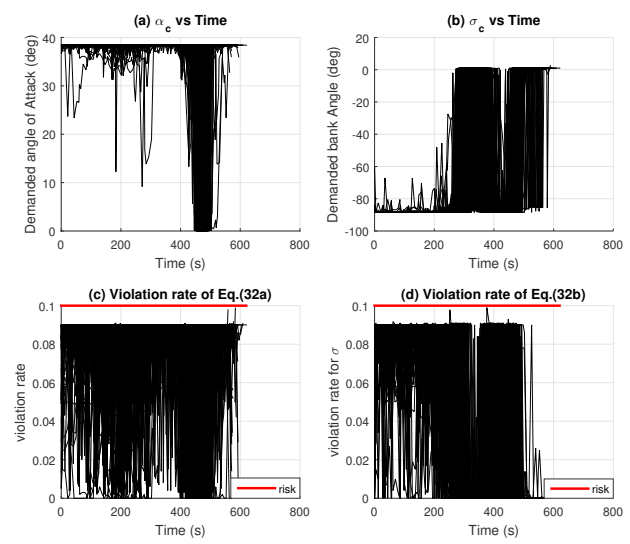

Fig. 5: Control and chance constraint results

It can be seen from Fig. 4 that the uncertain variables appearing in the dynamics and constraints cause some deviations of the optimal trajectory. But the optimal control structure can still be preserved. This can be observed from the demanded AOA and band angle ensembles shown in Fig.5(a) and Fig.5(b). In terms of the stochastic terminal state chance constraints given by Eq.(26), it is evaluated that all the violation rates corresponding to the terminal state chance constraints are less than the pre-assigned risk parameter. More 
precisely, the average violation rate values for constraint (26) are 0.043 and 0.046 , respectively.

Moreover, the stochastic control actuation constraints depicted in Fig.5(c) and Fig.5(d) convey that the actual violation rates for each individual trajectory are below the maximum allowable rate. The aggressive behaviour of the constraint violation trajectories, together with the switching control structure obtained, validates that each individual flight trajectory among the trajectory ensemble can be a near-optimal solution for the stochastic spacecraft entry problem under the consideration of control and terminal state probabilistic constraints. This further indicates that the deterministic CCSOCP framework constructed in Section III is well-suited for the newly developed hybrid optimal control solver.

\section{CONCLUding REMARKS}

In this paper, a deterministic CCSOCP framework which can generate near-optimal flight trajectories for the stochastic spacecraft entry trajectory planning problem with the consideration of chance constraints was presented. The proposed framework discretized the uncertain variables in the dynamics and constraints and created the trajectory ensemble such that the resulting discretized formulation can be tackled by standard optimal control solvers. To effectively calculate the gradient information and apply gradient-based optimization algorithms, a smooth and differentiable approximation function was applied to replace the chance constraints, thereby providing reliable gradient information. A detailed simulation study was carried out to illustrate the effectiveness and key features of the chance constraint handling strategy. In addition, the stochastic trajectory ensemble of the spacecraft entry problem was also generated by performing the proposed method to solve the chance-constrained model for noiseperturbed dynamical systems. The results demonstrated the advantages of the proposed method in terms of its reliability, optimality and conservatism.

In our follow-up work, we will extend the proposed CCSOCP optimization framework by exploring other types of uncertainty. For example, the system will be affected by a time-dependent random process rather than the random variable. Moreover, based on the obtained trajectory ensemble, we will develop a deep artificial neural network in order to achieve the optimal state feedback for the investigated problem.

\section{REFERENCES}

[1] S. Spedicato and G. Notarstefano, "Minimum-time trajectory generation for quadrotors in constrained environments," IEEE Transactions on Control Systems Technology, vol. PP, no. 99, pp. 1-10;, 2017.

[2] A. Rucco, G. Notarstefano, and J. Hauser, "An efficient minimumtime trajectory generation strategy for two-track car vehicles," IEEE Transactions on Control Systems Technology, vol. 23, no. 4, pp. 1505$1519,2015$.

[3] A. J. Hausler, A. Saccon, A. P. Aguiar, J. Hauser, and A. M. Pascoal, "Energy-optimal motion planning for multiple robotic vehicles with collision avoidance," IEEE Transactions on Control Systems Technology, vol. 24 , no. 3, pp. 867-883, 2016.

[4] B. A. Conway, "A survey of methods available for the numerical optimization of continuous dynamic systems," Journal of Optimization Theory and Applications, vol. 152, no. 2, pp. 271-306, 2012.

[5] D. Garg, M. Patterson, W. W. Hager, A. V. Rao, D. A. Benson, and G. T. Huntington, "A unified framework for the numerical solution of optimal control problems using pseudospectral methods," Automatica, vol. 46, no. 11, pp. 1843-1851, 2010.

[6] R. Padhi, S. N. Balakrishnan, and T. Randolph, "Adaptive-critic based optimal neuro control synthesis for distributed parameter systems," Automatica, vol. 37, no. 8, pp. 1223-1234, 2001.

[7] V. Yadav, R. Padhi, and S. N. Balakrishnan, "Robust/optimal temperature profile control of a high-speed aerospace vehicle using neural networks," IEEE Transactions on Neural Networks, vol. 18, no. 4, pp. 1115-1128, 2007.
[8] G. Misra and X. Bai, "Task-constrained trajectory planning of freefloating space-robotic systems using convex optimization," Journal of Guidance, Control, and Dynamics, vol. 40, no. 11, pp. 2857-2870, 2017.

[9] M. Pontani and B. A. Conway, "Minimum-fuel finite-thrust relative orbit maneuvers via indirect heuristic method," Journal of Guidance, Control, and Dynamics, vol. 38, no. 5, pp. 913-924, 2014.

[10] J. A. Englander and B. A. Conway, "Automated solution of the lowthrust interplanetary trajectory problem," Journal of Guidance, Control, and Dynamics, vol. 40, no. 1, pp. 15-27, 2017.

[11] D. Gonzalez-Arribas, M. Soler, and M. Sanjurjo-Rivo, "Robust aircraft trajectory planning under wind uncertainty using optimal control," Journal of Guidance, Control, and Dynamics, pp. 1-16, 2017.

[12] Z. Zhao and M. Kumar, "Split-bernstein approach to chance-constrained optimal control," Journal of Guidance, Control, and Dynamics, vol. 40, no. 11, pp. 2782-2795, 2017.

[13] J. Xu, P. Shi, C. Lim, C. Cai, and Y. Zou, "Reliable tracking control for under-actuated quadrotors with wind disturbances," IEEE Transactions on Systems, Man, and Cybernetics: Systems, vol. PP, no. 99, pp. 1-12, 2018.

[14] M. Lorenzen, F. Dabbene, R. Tempo, and F. Allgower, "Constrainttightening and stability in stochastic model predictive control," IEEE Transactions on Automatic Control, vol. 62, no. 7, pp. 3165-3177, 2017.

[15] R. Padhi and S. Balakrishnan, "Optimal dynamic inversion control design for a class of nonlinear distributed parameter systems with continuous and discrete actuators," IET Control Theory \& Applications, vol. 1, no. 6, pp. 1662-1671, 2007.

[16] X. Cao, P. Shi, Z. Li, and M. Liu, "Neural-network-based adaptive backstepping control with application to spacecraft attitude regulation," IEEE Transactions on Neural Networks and Learning Systems, vol. 29, no. 9, pp. 4303-4313, 2018.

[17] Y. Huang, Y. Zhang, P. Shi, Z. Wu, J. Qian, and J. A. Chambers, "Robust kalman filters based on gaussian scale mixture distributions with application to target tracking," IEEE Transactions on Systems, Man, and Cybernetics: Systems, vol. PP, no. 99, pp. 1-15, 2018.

[18] S. Salomon, G. Avigad, P. J. Fleming, and R. C. Purshouse, "Active robust optimization: Enhancing robustness to uncertain environments," IEEE Transactions on Cybernetics, vol. 44, no. 11, pp. 2221-2231, 2014.

[19] M. Cannon, Q. Cheng, B. Kouvaritakis, and S. V. Rakovic, "Stochastic tube mpc with state estimation," Automatica, vol. 48, no. 3, pp. 536-541, 2012.

[20] A. Geletu, M. Kloppel, A. Hoffmann, and P. Li, "A tractable approximation of non-convex chance constrained optimization with non-gaussian uncertainties," Engineering Optimization, vol. 47, no. 4, pp. 495-520, 2015.

[21] A. Geletu, A. Hoffmann, M. Kloppel, and P. Li, "An inner-outer approximation approach to chance constrained optimization," SIAM Journal on Optimization, vol. 27, no. 3, pp. 1834-1857, 2017.

[22] L. Blackmore, M. Ono, and B. C. Williams, "Chance-constrained optimal path planning with obstacles," IEEE Transactions on Robotics, vol. 27, no. 6, pp. 1080-1094, 2011.

[23] P. Dutta and R. Bhattacharya, "Nonlinear estimation of hypersonic state trajectories in bayesian framework with polynomial chaos," Journal of Guidance, Control, and Dynamics, vol. 33, no. 6, pp. 1765-1778, 2010.

[24] I. Dimov, Monte Carlo methods for applied scientists. World Scientific, 2008.

[25] A. Nemirovski and A. Shapiro, "Convex approximations of chance constrained programs," SIAM Journal on Optimization, vol. 17, no. 4, pp. 969-996, 2006

[26] C. Feng, F. Dabbene, and C. M. Lagoa, "A kinship function approach to robust and probabilistic optimization under polynomial uncertainty," IEEE Transactions on Automatic Control, vol. 56, no. 7, pp. 1509-1523, 2011.

[27] R. Chai, A. Savvaris, and A. Tsourdos, "Violation learning differential evolution-based $\mathrm{hp}$-adaptive pseudospectral method for trajectory optimization of space maneuver vehicle," IEEE Transactions on Aerospace and Electronic Systems, vol. 53, no. 4, pp. 2031-2044, 2017.

[28] R. Chai, A. Savvaris, A. Tsourdos, S. Chai, and Y. Xia, "Trajectory optimization of space maneuver vehicle using a hybrid optimal control solver," IEEE Transactions on Cybernetics, vol. PP, no. 99, pp. 1-14, 2017.

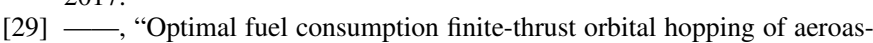
sisted spacecraft," Aerospace Science and Technology, vol. 75, pp. 172182,2018 
2019-04-18

Stochastic spacecraft trajectory optimization with the consideration of chance constraints

\author{
Chai, Runqi
}

IEEE

Chai R, Savvaris A, Tsourdos A, et al., (2020) Stochastic spacecraft trajectory optimization with the consideration of chance constraints. IEEE Transactions on Control Systems Technology, Volume 28, Issue 4, July 2020, pp. 1550-1559

https://doi.org/10.1109/TCST.2019.2908938

Downloaded from Cranfield Library Services E-Repository 